\title{
TU/e Emonowe

\section{Ageing-aware charging of lithium-ion batteries using an electrochemistry-based model with capacity-loss side reactions}

\section{Citation for published version (APA):}

Khalik, Z., Bergveld, H. J., \& Donkers, M. C. F. (2020). Ageing-aware charging of lithium-ion batteries using an electrochemistry-based model with capacity-loss side reactions. In 2020 American Control Conference, ACC 2020 (pp. 2213-2218). [9147404] (Proceedings of the American Control Conference; Vol. 2020-July). Institute of Electrical and Electronics Engineers. https://doi.org/10.23919/ACC45564.2020.9147404

DOI:

10.23919/ACC45564.2020.9147404

Document status and date:

Published: 01/07/2020

\section{Document Version:}

Accepted manuscript including changes made at the peer-review stage

\section{Please check the document version of this publication:}

- A submitted manuscript is the version of the article upon submission and before peer-review. There can be important differences between the submitted version and the official published version of record. People interested in the research are advised to contact the author for the final version of the publication, or visit the DOI to the publisher's website.

- The final author version and the galley proof are versions of the publication after peer review.

- The final published version features the final layout of the paper including the volume, issue and page numbers.

Link to publication

\footnotetext{
General rights

- You may freely distribute the URL identifying the publication in the public portal. follow below link for the End User Agreement:

www.tue.nl/taverne

\section{Take down policy}

If you believe that this document breaches copyright please contact us at:

openaccess@tue.nl

providing details and we will investigate your claim.
}

Copyright and moral rights for the publications made accessible in the public portal are retained by the authors and/or other copyright owners and it is a condition of accessing publications that users recognise and abide by the legal requirements associated with these rights.

- Users may download and print one copy of any publication from the public portal for the purpose of private study or research.

- You may not further distribute the material or use it for any profit-making activity or commercial gain

If the publication is distributed under the terms of Article $25 \mathrm{fa}$ of the Dutch Copyright Act, indicated by the "Taverne" license above, please 


\title{
Ageing-Aware Charging of Lithium-ion Batteries Using an Electrochemistry-Based Model with Capacity-Loss Side Reactions
}

\author{
Z. Khalik H.J. Bergveld M.C.F. Donkers
}

\begin{abstract}
In this paper, we utilize a Doyle-Fuller-Newman (DFN) model including capacity-loss side reactions to present a model-based design method for multi-stage charging protocols. This design method allows making a trade-off between charging time and battery ageing in a more systematic way. The results are leveraged by a highly efficient implementation of the DFN model, that has a short computation time. We show that by obtaining the Pareto front that describes the optimal trade-off between charging time and battery ageing for a single cycle, the results can be extended to the lifetime of the battery. Finally we show that the negative electrode over-potential is not always a good indicator for ageing, and that ageing will occur even when the battery operates in over-potential regions that are considered to not lead to ageing.
\end{abstract}

\section{INTRODUCTION}

Lithium-ion batteries are commonly charged using a socalled constant-current-constant-voltage (CC-CV) protocol, see e.g. [1], where the battery is initially charged at a set constant current, followed by a phase where the battery is kept at a constant voltage, until the current drops to a certain value. This current limit and voltage limit are determined by a trade-off between a short charging time and long cycle life. Indeed, besides the main chemical reaction, i.e., the one that stores energy in the battery, several side reactions occur that eventually lead to capacity fade and power fade [2]. Shortening the charging time without affecting the longevity of the battery is of interest, particularly for electric vehicles, where the relatively long charging times (when compared to refueling time of a conventional vehicle) are considered to be problematic.

Optimizing the charging protocol to achieve short charging times and limited ageing has received ample attention in both the electrochemical as well as the control systems literature. A traditional approach has been to improve on the CC-CV protocol by introducing additional $\mathrm{CC}$ or $\mathrm{CV}$ stages, e.g., the CV-CC-CV protocol [1], or the CC-CC-CV protocol [3]. However, the selection of current and voltage limits is not trivial. To find the limits that provide a good trade-off between battery ageing and charging time, many experiments need to be performed, see e.g., [4].

This work has received financial support from the Horizon 2020 programme of the European Union under the grants 'Electric Vehicle Enhanced Range, Lifetime And Safety Through INGenious battery management' (EVERLASTING-713771) and 'Advancing fail-aware, fail-safe, and failoperational electronic components, systems, and architectures for fully automated driving to make future mobility safer, affordable, and end-user acceptable' (AutoDrive-737469).

The authors are with the Dept. of Electrical Eng. of Eindhoven University of Technology, Netherlands. Henk Jan Bergveld is also affiliated with NXP Semiconductors, Eindhoven, Netherlands. E-mail: $\{z . k h a l i k$, h.j.bergveld,m.c.f.donkers\}@tue.nl
A more recent trend is to use model-based methods to systematically make the trade-off between battery ageing and charging time. The models used in these papers can vary from empirical models, such as the equivalent-circuit model (ECM) [5] to electrochemistry-based models that describe the main electrochemical reaction (i.e., the reaction that leads to storing energy) such as the Doyle-Fuller-Newman (DFN) model [6],see, e.g., [7] or the single-particle model (SPM), see, e.g., [8]. However, since these models do not describe the ageing of the battery, these papers rely on estimated states obtained through battery models, and define regions based on these states that should be avoided in order to not promote excessive ageing. These regions are incorporated as constraints in the optimization problem, and show the potential of model-based control for ageing-aware charging.

Of course, ageing can never be prevented and the transition between excessive and non-excessive ageing is not necessarily a sharp transition. Therefore, alongside this approach, there has been attention into incorporating ageing models into the optimization problem. These ageing models can be empirical as done in, e.g., [9], which are used in conjunction with ECMs, or they can be electrochemical, such as the sidereaction model introduced in [10], which can be incorporated into the DFN model. Some results using such side-reaction models exist in literature, e.g., [11], which uses external toolboxes such as CasADi [12] for simulation. However, the use of such toolboxes does not necessarily provide a computationally efficient model, which restricts the prediction horizon in the model-predictive control approach taken in [11].

In this paper, we present a model-based design method for multi-stage charging protocols, where this leads to a trade-off between charging time and ageing. The results are leveraged by the recent development of a highly efficient implementation of the DFN model [13]. This highly efficient model has low computation times, which allows this model to be used in model-based optimization methods. While we will focus on multi-stage charging protocols in this paper, extensions to full model-based charging can be done based on the model implementation used in this paper. Finally, the paper shows that the negative electrode over-potential is not always a good indicator for ageing, and that an effective trade-off between charging time and ageing cannot be made by merely setting constraints on this over-potential.

\section{BATTERY MODELING}

In this section, we briefly formulate the Doyle-FullerNewman (DFN) model [6], including the modeling of 


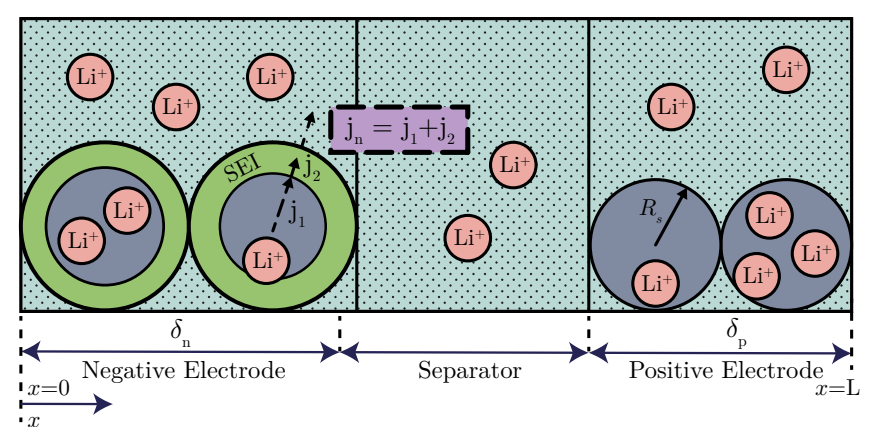

Fig. 1: DFN modeling approach for a Li-ion cell.

capacity-loss side reactions. The model represents the side reactions, in which Li-ions are consumed to form an SEI layer at the negative electrode [10]. Furthermore, we will formulate the equations that describe the ageing model, which is used to compute the capacity of an aged cell. The implementation of the model is similar to the implementation presented in [13], and is omitted in this paper owing to space limitations.

\section{A. Doyle-Fuller-Newman Model With Capacity-Loss Side Reactions}

The DFN model is a widely used electrochemistry-based model introduced in [6]. Fig. 1 illustrates the modeling approach for a Li-ion cell. In the $x$ dimension, the cell is divided into three regions, namely the negative electrode, the separator, and the positive electrode. The DFN model describes the potential and concentration gradients in the solid and electrolyte phase that result from the main electrochemical reaction, i.e., the reaction that leads to storing energy. In the considered side-reaction model [10], the particles in the negative electrode have a so-called solid-electrolyte interface (SEI) layer, which is formed at the first charge of the battery. During the operation of the cell over its lifetime, side reactions consume $\mathrm{Li}$-ions, which leads to a growth of the SEI. This consumption of Li-ions leads to capacity fade of the cell, while the build-up of the resistive SEI layer leads to power fade of the cell. We will shortly summarize the governing equations of the DFN model considering side reactions. The formulation of the DFN model will be mostly based on [14], while the side-reactions model is based on [10]. For compactness of notation, the time and space dependency of the variables given will be left out of the equations.

The DFN model is governed by four coupled partial differential equations (PDEs):

1) The Li-ion concentration in the solid phase $c_{s}(x, r, t)$ for $x \in\left[0, \delta_{n}\right] \cup\left[L-\delta_{p}, L\right]$ is given by Fick's law as

$$
\frac{\partial c_{s}}{\partial t}=\frac{D_{s}}{r^{2}} \frac{\partial}{\partial r}\left(r^{2} \frac{\partial c_{s}}{\partial r}\right) \text {, }
$$

with boundary conditions

$$
\left.\frac{\partial c_{s}}{\partial r}\right|_{r=0}=0, \quad-\left.D_{s} \frac{\partial c_{s}}{\partial r}\right|_{r=R_{s}}=j_{1},
$$

with $D_{s}$ the diffusion coefficient of lithium in the solid phase, and $j_{1}(x, t)$ is the molar flux of Li-ions deintercalating out of the solid phase. Furthermore, $L$, and $\delta_{n}$, and $\delta_{p}$ are the thickness of the cell, and the negative and positive electrode, respectively (see Fig. 1).

2) The Li-ion concentration in the electrolyte phase $c_{e}(x, t)$ for $x \in[0, L]$ is given by

$$
\varepsilon_{e} \frac{\partial c_{e}}{\partial t}=\frac{\partial}{\partial x}\left(D_{e} \frac{\partial c_{e}}{\partial x}\right)+a_{s}\left(1-t_{+}^{0}\right) j_{n},
$$

with boundary conditions

$$
\left.D_{e}^{\mathrm{eff}} \frac{\partial c_{e}}{\partial x}\right|_{x=0}=\left.D_{e}^{\mathrm{eff}} \frac{\partial c_{e}}{\partial x}\right|_{x=L}=0
$$

where $j_{n}=j_{1}+j_{2}$ is the net molar flux exiting the particle, in which $j_{2}$ is the side-reaction flux of Li-ions being consumed by the SEI layer. Note that this paper considers only SEI build up in the negative electrode, and therefore in the separator and in the positive electrode $j_{2}=0$. Furthermore, $D_{e}^{\mathrm{eff}}=D_{e} \varepsilon_{e}^{p}$ is the effective Li-ion diffusion coefficient in the electrolyte phase, in which $\varepsilon_{e}$ is the electrolyte phase volume fraction, $p$ is the Bruggeman porosity exponent, and $D_{e}$ is the diffusion constant of $\mathrm{Li}$-ions in the electrolyte. Finally, in (2), $a_{s}=3 \varepsilon_{s} / R_{s}$ is the specific interfacial surface area, in which $\varepsilon_{s}$ is the active material volume fraction, and $t_{+}^{0}$ is the transference number of $\mathrm{Li}$ ions.

3) The potential in the solid phase $\phi_{s}(x, t)$ for $x \in\left[0, \delta_{n}\right] \cup$ $\left[L-\delta_{p}, L\right]$ is given by Ohm's law, i.e.,

$$
\frac{\partial}{\partial x}\left(\sigma^{\mathrm{eff}} \frac{\partial \phi_{s}}{\partial x}\right)=a_{s} F j_{n},
$$

with boundary conditions

$$
\begin{aligned}
& \left.\sigma^{\text {eff }} \frac{\partial \phi_{s}}{\partial x}\right|_{x=0}=\frac{i_{\text {app }}}{A_{\text {surf }}},\left.\quad \sigma^{\text {eff } \frac{\partial \phi_{s}}{\partial x}}\right|_{x=\delta_{n}}=0 \\
& \left.\sigma^{\text {eff }} \frac{\partial \phi_{s}}{\partial x}\right|_{x=L-\delta_{p}}=0,\left.\quad \sigma^{\text {eff }} \frac{\partial \phi_{s}}{\partial x}\right|_{x=L}=\frac{i_{\text {app }}}{A_{\text {surf }}},
\end{aligned}
$$

where $F$ is Faraday's constant, $\sigma^{\text {eff }}=\varepsilon_{s} \sigma$ is the effective electronic conductivity of a porous electrode, in which $\sigma$ is the conductivity of the solid material, $A_{\text {surf }}$ is the area of the electrode plate, and $i_{\text {app }}(t)$ is the applied current through the battery, with $i_{\text {app }}>0$ indicating charging.

4) The potential in the electrolyte phase $\phi_{e}(x, t)$ for $x \in$ $[0, L]$ is given by

$$
\frac{\partial}{\partial x}\left(\kappa_{\mathrm{eff}} \frac{\partial \phi_{e}}{\partial x}+\kappa_{\mathrm{eff}} \frac{2 R T}{F}\left(t_{+}^{0}-1\right) \frac{\partial \ln c_{e}}{\partial x}\right)=-a_{s} F j_{n},
$$

with boundary conditions

$$
\left.\kappa_{\text {eff }} \frac{\partial \phi_{e}}{\partial x}\right|_{x=0}=\left.\phi_{e}\right|_{x=L}=0,
$$

in which $\kappa_{\text {eff }}=\kappa \varepsilon_{e}^{p}$ is the effective ionic conductivity, where $\kappa$ is the ionic conductivity of the electrolyte, $R$ is the universal gas constant, and $T$ is the absolute temperature.

The above PDEs (1)-(4) can describe the terminal voltage, which is given by

$$
V(t)=\phi_{s}(L, t)-\phi_{s}(0, t) .
$$




\section{B. Rate Equation for Main \& Side Reaction}

The above PDEs (1)-(4) are coupled by a Butler-Volmer rate equation $j_{n}$, which describes the net chemical reaction rate at the solid-electrolyte interface. For the main intercalation reaction, i.e., the reaction that leads to storing energy, the rate equation is given by

$$
j_{1}=\frac{i_{0,1}}{F}\left(\exp \left(\frac{\alpha_{a} F}{R T} \eta_{1}\right)-\exp \left(-\frac{\alpha_{c} F}{R T} \eta_{1}\right)\right)
$$

which is only defined for $x \in\left[0, \delta_{n}\right] \cup\left[L-\delta_{p}, L\right]$ and assumed zero for $x \in\left(\delta_{n}, L-\delta_{p}\right)$. In (6a), $\alpha_{a}$ is the anodic transfer coefficient, $\alpha_{c}$ is the cathodic transfer coefficient, and the over-potential at the electrodes $\eta_{1}(x, t)$ is defined as

$$
\eta_{1}=\phi_{s}-\phi_{e}-U\left(\bar{c}_{s}\right)-F R_{f} j_{n}
$$

in which $U\left(\bar{c}_{s}\right)$ denotes the equilibrium potential of the electrode, which can be given by a pre-defined function typically of the solid-phase concentration at the SEI $\bar{c}_{s}(x, t)=$ $c_{s}\left(R_{s}, x, t\right)$, and $R_{f}$ is the film resistance given by

$$
\frac{\partial R_{f}}{\partial t}=-\frac{\tilde{V}_{f}}{\sigma_{f}} j_{2}
$$

with initial condition $R_{f}(x, 0)=R_{f, 0}$, and where $\tilde{V}_{f}$ is the molar volume and $\sigma_{f}$ is the conductivity of the SEI. Furthermore, the exchange current density $i_{0,1}$ in (6a) is given by

$$
i_{0,1}=k_{0} c_{e}^{\alpha_{a}}\left(c_{s, \max }-\bar{c}_{s}\right)^{\alpha_{a}} \bar{c}_{s}^{\alpha_{c}}
$$

where $k_{0}$ is the rate constant of the electrochemical reaction, and $c_{s, \max }$ is the maximum concentration in the solid-phase.

The chemical reaction rate of the side reactions that consume Li-ions is described by a Tafel equation of the form

$$
j_{2}=-\frac{i_{0,2}}{F} \exp \left(-\frac{2 \alpha_{c, 2} F}{R T} \eta_{2}\right),
$$

where $i_{0,2}$ is the exchange current density of the side reaction, which is assumed to be a constant, and $\eta_{2}$ is the side reaction over-potential given by

$$
\eta_{2}=\phi_{s}-\phi_{e}-U_{2}-F R_{f} j_{n}
$$

in which $U_{2}$ is the equilibrium potential of the side reactions, which is usually assumed to be a constant.

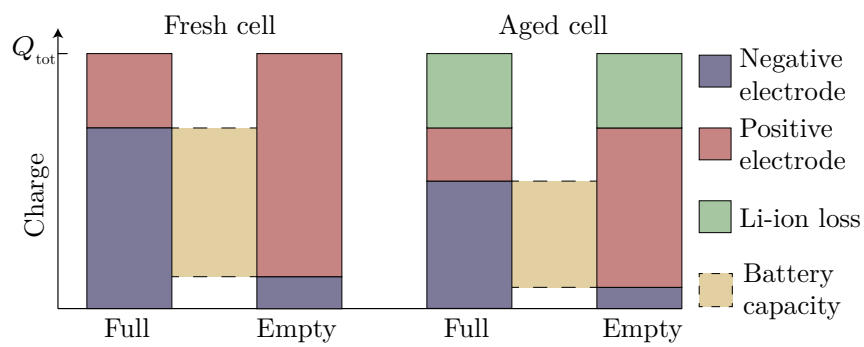

Fig. 2: Schematic representation of the ageing model.

\section{Ageing model}

The amount of charge lost due to loss of Li-ions as a result of side reactions can be computed as [10]

$$
\frac{d Q_{\text {ion, loss }}}{d t}=-a_{s} A_{\text {surf }} F \int_{0}^{\delta_{n}} j_{2} d x .
$$

The Li-ion loss does not directly result into loss of battery capacity [15]. To see this, it should be noted that the total amount of charge stored in a fresh cell is given by

$$
Q_{\mathrm{tot}}=w_{\max } \gamma_{n}+z_{\min } \gamma_{p}=w_{\min } \gamma_{n}+z_{\max } \gamma_{p},
$$

where $\gamma_{i}=\varepsilon_{s, i} \delta_{i} c_{\mathrm{s}, \max , \mathrm{i}} A_{\text {surf }} F$, for $i \in\{n, p\}$, where $n$ and $p$ denote the values of the parameters at the negative and postive electrode, respectively. Furthermore, $w$ and $z$ denote the stoichiometric values at the negative and positive electrode, respectively, while the subscripts min and max indicate the minimum and maximum values of $w$ and $z$, respectively, for a fresh cell. The stoichiometric values at $0 \%$ and $100 \%$ for an aged battery must satisfy

$$
\begin{array}{r}
w_{0} \gamma_{n}+z_{0} \gamma_{p}=Q_{\mathrm{tot}}-Q_{\text {ion,loss }} \\
w_{100} \gamma_{n}+z_{100} \gamma_{p}=Q_{\mathrm{tot}}-Q_{\text {ion,loss }}
\end{array}
$$

Note that for a fresh cell, $w_{0}, w_{100}, z_{0}, z_{100}$ correspond to $w_{\min }, w_{\max }, z_{\max }, z_{\min }$, respectively. Furthermore, since $0 \%$ state-of-charge (SOC) and $100 \%$ SOC are defined at specified voltages, the equilibrium potentials must satisfy

$$
\begin{array}{r}
U_{p}\left(z_{0}\right)-U_{n}\left(w_{0}\right)=V_{\min } \\
U_{p}\left(z_{100}\right)-U_{n}\left(w_{100}\right)=V_{\max },
\end{array}
$$

where $V_{\max }=U_{p}\left(z_{\min }\right)-U_{n}\left(w_{\max }\right)$, and $V_{\min }=$ $U_{p}\left(z_{\max }\right)-U_{n}\left(w_{\min }\right)$. By solving $(8)$, solutions for $w_{0}$, $w_{100}, z_{0}$, and $z_{100}$ are obtained. These solutions can be used to compute the degraded capacity of the cell $Q_{\text {bat }}$, i.e.,

$$
Q_{\mathrm{bat}}=\left(w_{100}-w_{0}\right) \gamma_{n}=\left(z_{0}-z_{100}\right) \gamma_{p} .
$$

How the ageing reaction affects the battery capacity is illustrated in Fig. 2. In this figure, it can be seen that for an aged cell, the charge in the positive and negative electrodes decrease as Li-ions are lost, which leads to a decrease in battery capacity. The capacity drop of the battery, however, is not necessarily equal to the amount of Li-ions consumed by the side reaction, as the side reaction also leads to a shift in the equilibrium potential of the electrodes.

TABLE I: Parameters for the side-reaction model. Parameters $U_{2}$ and $\sigma_{f}$ have been extracted from [15].

\begin{tabular}{lll} 
Symbol & Unit & Value \\
\hline$i_{0,2}$ & {$\left[A m^{-2}\right]$} & $1.178 \times 10^{-7}$ \\
$U_{2}$ & {$[V]$} & 0.21 \\
$\tilde{V}_{f}$ & {$\left[\mathrm{~m}^{3} \mathrm{~mol}^{-1}\right]$} & $1 \times 10^{-5}$ \\
$\sigma_{f}$ & {$\left[\Omega^{-1} \mathrm{~m}^{-1}\right]$} & $2.3 \times 10^{-6}$ \\
$R_{f, 0}$ & {$\left[\Omega m^{2}\right]$} & $5.5 \times 10^{-3}$
\end{tabular}




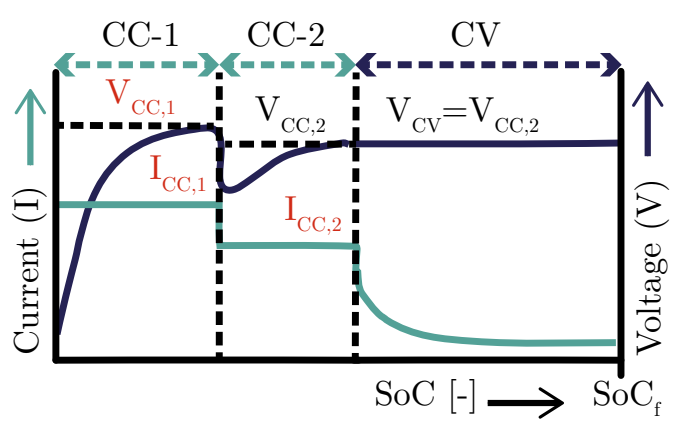

Fig. 3: The 2-CC-CV(3) protocol shown as an example of a multi-stage charging protocol used for simulation. The redcolored variables indicate the design-adjustable parameters.

\section{Ageing-Aware Charging Protocol Design}

In this section, we will study the impact of several charging strategies on battery ageing. First, we will study ageing over a single cycle, to determine the Pareto optimal set of parameters of several charging strategies that lead to the ideal trade-off that can be made between charging time and battery ageing. Then, we will study ageing over a long period, by simulating the Pareto optimal set of parameters over the lifetime of the battery. Finally, we investigate the use of over-potential information to limit battery ageing. The parameters of the main reaction model (including the electrode equilibrium potential expressions) are taken from [12]. Note that this set of parameters represents a highenergy cell, for which it is generally known that the minimum charging time that can be achieved is generally higher than for a high-power cell. The parameters of the side-reaction model have been selected such that a somewhat realistic amount of ageing is observed, i.e., $80 \%$ of the capacity is left after around 600 charge-discharge cycles. These sidereaction model parameters have been listed in Table I.

\section{A. Charging protocols}

The simulation studies have been done using a CC-CV (Constant-Current-Constant-Voltage) charging protocol and several multi-stage charging protocols. Note that, for the remainder of this paper, the following notation will be used to indicate the multi-stage charging protocols: $N-\mathrm{CC}-\mathrm{CV}(D)$, where $N$ denotes the number of $C C$ stages, and $D$ denotes the amount of design-adjustable variables. As an example of a multi-stage charging protocol, the 2-CC-CV(3) charging protocol is shown in Fig. 3. In this protocol, the battery is first charged with a constant current $I_{\mathrm{CC}, 1}$ until a voltage $V_{\mathrm{CC}, 1}$ is reached. Then, the battery is charged with a constant current $I_{\mathrm{CC}, 2}$ until the next voltage limit $V_{\mathrm{CC}, 2}$ is reached. Finally, a CV stage follows where the battery is kept at a constant voltage $V_{\mathrm{CV}}$ until a final state-of-charge $\mathrm{SOC}_{f}$ is reached. For this protocol there are three design-adjustable parameters, namely $I_{\mathrm{CC}, 1}, I_{\mathrm{CC}, 2}$, and $V_{\mathrm{CC}, 1}$. The value for $V_{\mathrm{CC}, 2}=V_{\mathrm{CV}}$ is chosen as $V_{\max }$ in (8c) for a fresh cell, and $\mathrm{SOC}_{f}$ is fixed to $\mathrm{SOC}_{f}=95 \%$. Note that, for the remainder of the paper, we constraint $V_{\mathrm{CV}}=V_{\mathrm{CC}, n}$, where $n$ indicates the last CC stage. Further note that we use SOC to terminate the charging algorithm to ensure a fair comparison between

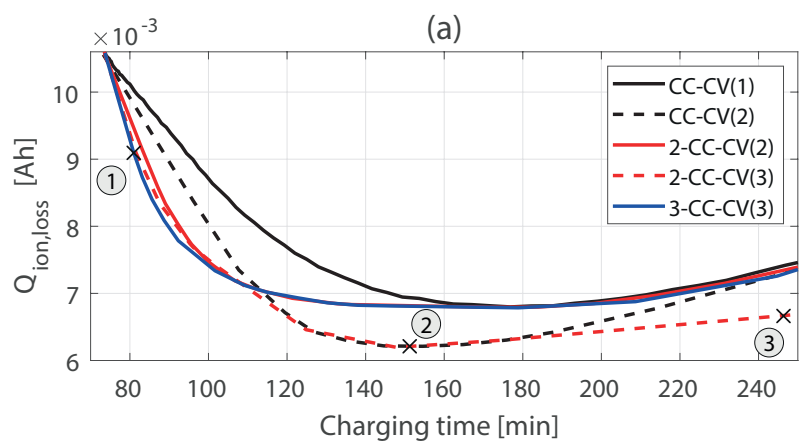

(b)

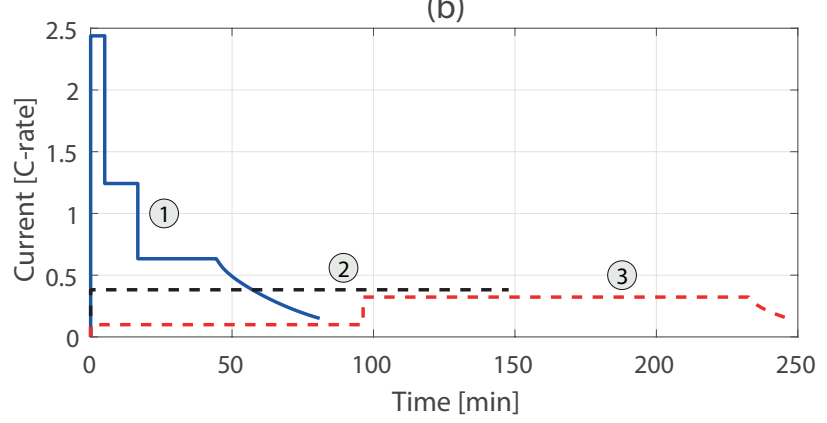

Fig. 4: In (a), a comparison of degradation vs charging time of several charging protocols is shown. In (b), selected current profiles at several points on (a) are shown.

different charging profiles, while in practice generally the $\mathrm{CV}$ stage is terminated when the current drops below a certain value $I_{\min }$. The CC-CV charging protocol is defined in a similar way, except that there is only one CC stage, and therefore the only design-adjustable parameter is $I_{\mathrm{CC}, 1}$. Other charging strategies that are considered in this paper are the CC-CV(2), 2-CC-CV(2), and 3-CC-CV(3). For CC$\mathrm{CV}(2)$, the design-adjustable parameters are the $\mathrm{CC}$ current $I_{\mathrm{CC}, 1}$ and the $\mathrm{CV}$ voltage $V_{\mathrm{CV}}$. For 2-CC-CV(2), the design adjustable parameters are $\mathrm{CC}$ currents $I_{\mathrm{CC}, 1}$ and $I_{\mathrm{CC}, 2}$. Finally, for 3-CC-CV(3), the design-adjustable parameters are $\mathrm{CC}$ currents $I_{\mathrm{CC}, 1}, I_{\mathrm{CC}, 2}$, and $I_{\mathrm{CC}, 3}$. Note that in the case of 2-CC-CV(2) and 3-CC-CV(3), the voltage limits, e.g. $V_{\mathrm{CC}, 1}$, are fixed as $V_{\max }$.

\section{B. Pareto Optimal Front for a Single Cycle}

In Fig. 4 (a), the degradation of the cell, in terms of Liions lost, i.e., $Q_{\text {ion,loss }}$, is plotted against charging time, for the CC-CV and multi-stage charging protocols. In order to obtain these results, the design-adjustable parameters for the charging protocols have been varied over a range of values. Specifically, the CC-stage currents have been varied in the range of $0.1 \mathrm{C}$ to $2.5 \mathrm{C}$, and the voltage limits have been varied in the range of $3.7 \mathrm{~V}$ to $4.4 \mathrm{~V}$, and in the case of $\mathrm{CC}-\mathrm{CV}(2), V_{\mathrm{CV}}$ has been varied in the range of $4.125 \mathrm{~V}$ to $4.4 \mathrm{~V}$. The curves in Fig. 4 are then obtained by taking the minimal degradation for a given charging time, for each charging protocol. Note that for the CC-CV protocol, since there is only 1 design-adjustable parameter, all the simulated points have by definition the minimal degradation for a given charging time. Note that, as an example, the trade-off curve 
for the 3-CC-CV(3) was constructed using 8000 chargecycle simulations, while the other trade-off curves have been constructed using fewer simulations. As each charge cycle takes about 1 second to compute (on average), even the tradeoff curve for the 3-CC-CV(3) protocol can be computed within 2.5 hours, which shows the potential of the model implementation used from [13] for (off-line) model-based optimization.

In Fig. 4 (a), we can first observe that the degradation of the multi-stage protocols is always equal to or less than the degradation using the CC-CV protocol. This is to be expected, since multi-stage protocols are a generalization of the CC-CV protocol. Furthermore, we see that up to around 170 min charging time, the curves follow an intuitive trend, where the battery degradation becomes smaller as charging time increases. However, after around $170 \mathrm{~min}$ charging time, the opposite trend happens, where battery degradation increases with increasing charging time. This can be explained by the fact that the degradation is computed through an integral over time. Therefore, as charging time increases, the integral is taken over a longer time, leading to higher degradation.

The better performance at higher charging times in Fig. 4 (a), can be explained by observing the current shown in Fig. 4 (b) at point 2 . Here, we see that the battery is simply charged with a constant current rate until the desired SOC of $95 \%$ is reached. The CC-CV(2) and 2-CC-CV-(3) protocols allow this, since the voltage limit can be chosen sufficiently high such that it is never reached until the battery is full. Note that the CV stage in general takes a significant amount of time when charging, which explains why charging in CC stages as long as possible is effective.

Finally, if we observe point 3 in Fig. 4 (b), we can see why less degradation is achieved at high charging times. At high charging times, the effect of the integral in (7) dominates over the effect that lower degradation can be achieved by applying a high charging current at low voltages. Thus, in this instance the better strategy is to keep the current as low as possible, until the remaining charging time is 150 minutes, which is the highest charging time on the Pareto front of the $\mathrm{CC}-\mathrm{CV}$ (2) protocol, after which the battery is charged with, in this case, a constant current.

\section{Lifetime Degradation}

In Fig. 5 (a), the battery cycle life is plotted against the average charging time during the course of the lifetime of the cell. This result has been obtained by taking the Pareto optimal combinations found in Fig. 4, and simulating for each of these combinations over the lifetime of cell, i.e., when the capacity of the battery reaches $80 \%$ of the initial capacity. However, rather than performing a single continuous simulation, the simulations have been done for each cycle separately. Here, the stoichiometric values at $0 \%$ and $100 \%$ are computed and updated at each cycle using (8). This is mainly done for the reason that the difference in scale between $j_{1}$ and $j_{2}$ causes numerical inaccuracies which add up over the lifetime of the cell. Note that the results in Fig. 4 have been obtained using only charge cycles, while the (a)

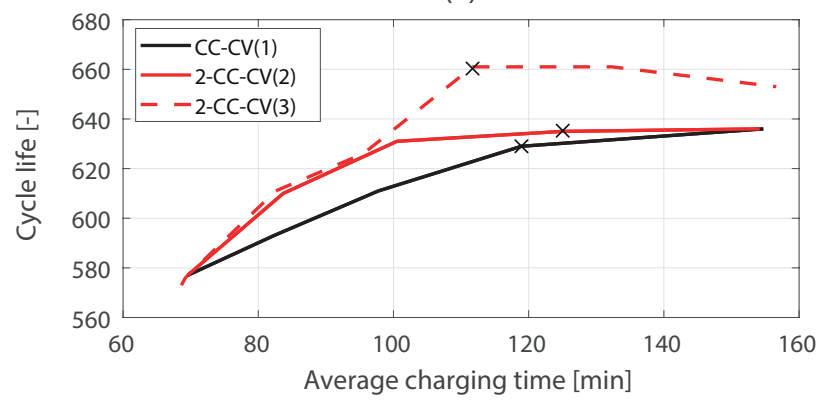

(b)

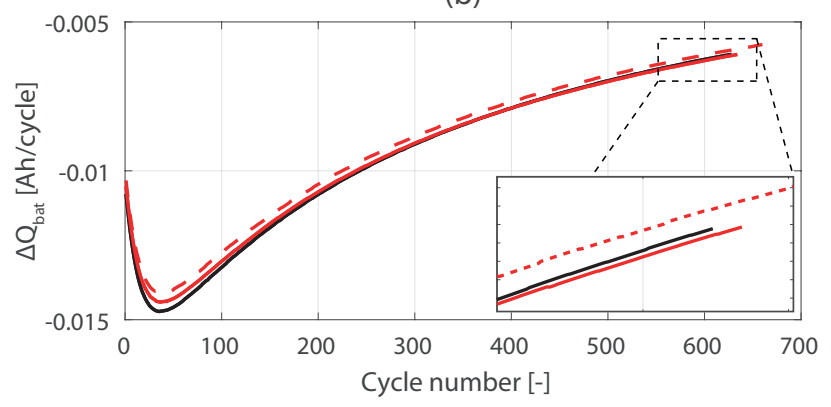

Fig. 5: In (a), cycle life vs average charging time for several charging protocols is shown. In (b), the differential capacity loss $\Delta Q_{\text {bat }}$ is shown. The crosses in (a) indicate at which charging time the curves in (b) are evaluated for.

results shown in fig. 5 have been obtained from dischargecharge cycles in order to represent actual use of the battery. The discharge cycle occurs at a rate of $-0.1 \mathrm{C}$, until a lower voltage limit $V_{\min }$, computed from (8c), is reached, after which the battery is charged.

As could be expected, the multi-stage charging protocols perform better than the CC-CV(1) protocol. Furthermore, the trends observed in Fig. 4 (a) are still visible, in which the performance of the 2-CC-CV(2) and 2-CC-CV(3) are almost equal up to around 100 minutes charging time, and the performance of the 2-CC-CV(3) protocol is much better than the 2-CC-CV(2) after around 100 minutes charging time. Therefore, we can conclude that the trends observed over a single cycle can be roughly extrapolated over the lifetime of the cell.

However, while the trends at the end-of-life of the cell are conserved, this is not necessarily true over the whole lifetime of the cell. In Fig. 5 (b), the differential capacity loss $\Delta Q_{\text {bat }}$ of the considered charging protocols at a charging time of around 130 minutes is shown over the lifetime of the cell. We can observe that the difference in differential capacity loss between the charging protocols varies over the lifetime of the cell. Until around 50 cycles, this difference gets larger, while after 50 cycles the difference becomes smaller. In the case of the difference between the CC-CV(1) and 2-CC-CV(2) protocol, after 250 cycles, this difference becomes visually indiscernible. This could suggest that when the battery ages, there is less to gain when choosing different charging strategies. However, if we observe the zoomed-in plot in Fig. 5 (b), we can also see that at some point, the 2-CC-CV(2) protocol actually has a higher $\Delta Q_{\text {bat }}$ compared 

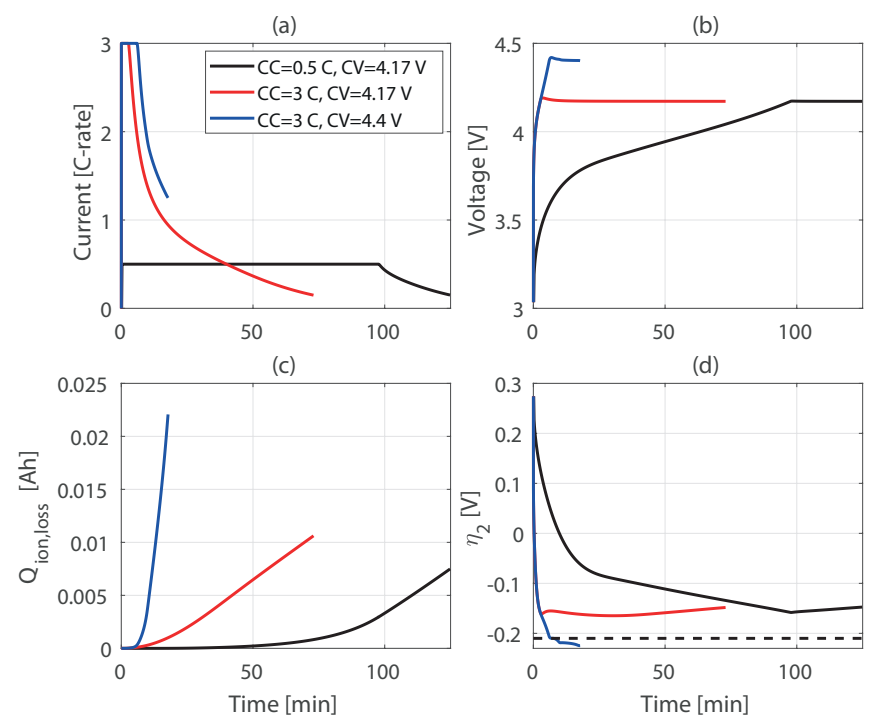

Fig. 6: Results showing the side-reaction over-potential $\eta_{2}$ for combinations of $\mathrm{CC}$ and $\mathrm{CV}$ levels with the CC-CV charging protocol, where (a), (b), (c), and (d) show the current, voltage, Li-ion loss $Q_{\text {ion,loss }}$, and $\eta_{2}$, respectively. The black dotted line indicates the points where $\eta_{2} \leq-U_{2}$.

to the CC-CV(1) protocol, while it had a lower $\Delta Q_{\text {bat }}$ than the CC-CV(1) in the beginning stages of its lifetime. This suggests then that even if a charging strategy is better than another for a fresh battery, it does not necessarily mean that it is better than the other strategy over the lifetime of the cell. Thus, we can conclude that in order to retain optimal performance over the lifetime of the cell, it is crucial to have a smart (model-based) charging strategy, which can adapt to the changing conditions of the cell over its lifetime.

\section{Side-Reaction Over-Potential as Ageing Indicator}

The side-reaction over-potential $\eta_{2}$ over time plotted for combinations of CC and CV levels can be seen in Fig. 6. We can see that as C-rates in the CC stage get larger, the over-potential becomes more negative, and as the $\mathrm{CV}$ voltage gets larger, the over-potential also becomes more negative. The constraint $\phi_{s}-\phi_{e}>0$ is often used to prevent lithium plating in the battery, e.g. in [7]. This constraint is equivalent to $\eta_{2}>-U_{2}$, which is the black dotted line shown in Fig. 6. However, from Fig. 6 (c), we can see that even at the highest $\mathrm{C}$-rate, where the minimum charging time is reached, this constraint is still not violated. The blue curve shows a scenario where this constraint is violated, which is at a combination of high currents and high voltages. From Fig. 6 (c), we can also see that when $\eta_{2} \leq-U_{2}$, the Liion loss increases significantly. Note that the blue curve is not actually fully charged, as the solid phase concentration became saturated before the battery could become fully charged. Of course, even in the other two cases of the black and red curves, even though the over-potential constraint is not violated, there is still a difference in how much the battery ages with each of the current profiles, as we can see from Fig. 6 (c). Still, it can serve as a hard constraint to prevent extreme scenarios that lead to lithium plating. However, as shown by the above analysis, side reaction over-potential cannot be used to make a trade-off between charging time and battery degradation effectively.

\section{CONCLUSIONS}

In this paper, we have utilized a Doyle-Fuller-Newman (DFN) model including capacity-loss side reactions to present a model-based design method for multi-stage charging protocols. This design method allows making a trade-off between charging time and battery ageing. The results have been leveraged by a highly efficient implementation of the DFN model, which has a short computation time, introduced in [13]. We have shown that by obtaining the Pareto front that describes the optimal trade-off between charging time and battery ageing for a single cycle, the results can be extended to the lifetime of the battery. Finally, we have shown that the negative electrode over-potential is not always a good indicator for ageing, and that ageing will occur even when the battery operates in over-potential regions that are considered to not lead to ageing.

\section{REFERENCES}

[1] P. Notten, J. O. h. Veld, and J. v. Beek, "Boostcharging Li-ion batteries: A challenging new charging concept," Journal of Power Sources, 2005.

[2] P. Arora, "Capacity Fade Mechanisms and Side Reactions in LithiumIon Batteries," Journal of The Electrochemical Society, 1998.

[3] D. Anseán, M. González, J. Viera, V. García, C. Blanco, and M. Valledor, "Fast charging technique for high power lithium iron phosphate batteries: A cycle life analysis," Journal of Power Sources, 2013.

[4] T. T. Vo, X. Chen, W. Shen, and A. Kapoor, "New charging strategy for lithium-ion batteries based on the integration of Taguchi method and state of charge estimation," Journal of Power Sources, 2015.

[5] Y. Gao, C. Zhang, Q. Liu, Y. Jiang, W. Ma, and Y. Mu, "An optimal charging strategy of lithium-ion batteries based on polarization and temperature rise," in Transportation Electrification Asia-Pacific (ITEC Asia-Pacific), 2014 IEEE Conference and Expo, 2014.

[6] M. Doyle, T. F. Fuller, and J. Newman, "Modeling of galvanostatic charge and discharge of the lithium/polymer/insertion cell," J. Electrochem. Soc., 1993.

[7] H. Perez, N. Shahmohammadhamedani, and S. Moura, "Enhanced Performance of Li-Ion Batteries via Modified Reference Governors and Electrochemical Models," IEEE/ASME Transactions on Mechatronics, 2015.

[8] C. Zou, X. Hu, Z. Wei, T. Wik, and B. Egardt, "Electrochemical Estimation and Control for Lithium-Ion Battery Health-Aware Fast Charging," IEEE Transactions on Industrial Electronics, 2018.

[9] H. E. Perez, X. Hu, S. Dey, and S. J. Moura, "Optimal Charging of LiIon Batteries With Coupled Electro-Thermal-Aging Dynamics," IEEE Trans. Veh. Technol, 2017.

[10] P. Ramadass, B. Haran, P. M. Gomadam, R. White, and B. N. Popov, "Development of First Principles Capacity Fade Model for Li-Ion Cells," Journal of The Electrochemical Society, 2004.

[11] S. Lucia, M. Torchio, D. M. Raimondo, R. Klein, R. D. Braatz, and R. Findeisen, "Towards adaptive health-aware charging of Li-ion batteries: A real-time predictive control approach using first-principles models," in American Control Conference (ACC), 2017, 2017.

[12] M. Torchio, L. Magni, R. B. Gopaluni, R. D. Braatz, and D. M. Raimondo, "LIONSIMBA: A matlab framework based on a finite novolume model suitable for Li-ion battery design, simulation, and control," J. Electrochem. Soc., 2016.

[13] Z. Khalik, H. J. Bergveld, and M. C. F. Donkers, "On Trade-offs Between Computational Complexity and Accuracy of Electrochemistrybased Battery Models," in Conf. Decision \& Control, 2019.

[14] K. A. Smith, C. D. Rahn, and C.-Y. Wang, "Control oriented 1d electrochemical model of lithium ion battery," Energy Convers. Manag., 2007

[15] R. Fu, S.-Y. Choe, V. Agubra, and J. Fergus, "Development of a physics-based degradation model for lithium ion polymer batteries considering side reactions," Journal of Power Sources, 2015. 